AMERICAN JOURNAL OF SOCIAL AND MANAGEMENT SCIENCES

ISSN Print: 2156-1540, ISSN Online: 2151-1559, doi:10.5251/ajsms.2010.1.2.164.180

(C) 2010, ScienceHuß, http://www.scihub.org/AJSMS

\title{
Anchoring and pre-existing knowledge in economic and financial settings
} Andrey Kudryavtsev and Gil Cohen

The Max Stern Academic College of Emek Yezreel, Emek Yezreel 19300, Israel

E-mail addresses: andreyk@yvc.ac.il, gilc@yvc.ac.il

\begin{abstract}
Contemporary research documents various psychological aspects of economic thought and decision-making. The main goal of our study is to analyze the role of the anchoring (bias) (Tversky and Kahneman, 1974) in perceiving economic and financial information, and, in particular, the effect of pre-existing knowledge on the degree of the bias. Anchoring bias refers to people's tendency to form their estimates for different categories, starting from a particular available, and often irrelevant, value and insufficiently adjusting their final judgments from this starting value. We carry out an experiment involving a group of MBA students, asking them to recall a number of recent economic and financial indicators (stock and bond market index returns, rates of inflation, currency exchange rates, etc.), with half of the participants receiving actual information about some unrelated indicators (anchors), before answering the questions. We document that significant anchoring bias is exhibited, on average, for each of our experimental questions and by vast majority of the participants, the degree of the bias being higher for women and older participants. Furthermore, in the context of the effect of pre-existing knowledge, we find that people exhibit significantly stronger bias for relatively more difficult questions. Our findings indicate that the less people know about an object or a category, the more they may be influenced by some, even arbitrary, anchors when estimating its characteristics or forecasting related future outcomes.
\end{abstract}

Keywords: Anchoring; Behavioral Economics and Finance; Experimental Economics and Finance; Information and Knowledge.

JEL Classification: D83, D89, G10.

\section{INTRODUCTION}

What do you expect the return on stock $X$ to be next year? Suppose that before answering this question you have just read a newspaper report indicating that the number of tourists visiting Japan increased by 10 percent over the last five years. Would this information influence your answer to the question about the stock return? Would your estimate be higher than if the newspaper report indicated that the number of tourists decreased by 10 percent?

When making judgments for which the answer is ambiguous, most people start with a particular value that is available to them, and then adjust their final judgment from this value, even if the starting value is entirely random. If this kind of adjustment is insufficient, then a phenomenon known as anchoring (or anchoring bias) occurs. Since first proposed by Tversky and Kahneman (1974), this phenomenon has been documented and studied extensively in various fields of knowledge, environments, and settings.

In this study, we analyze the effect of anchoring in perceiving economic and financial data. Particularly, we wish to shed light on the effect of pre-existing knowledge on the degree of the bias exhibited. We carry out an experiment involving a group of MBA students, asking them to recall a number of recent economic and financial indicators for Israeli economy, in general (rates of inflation, interest rate of the Bank of Israel, currency exchange rates), and Tel Aviv Stock Exchange, in particular (stock and bond market index values and returns, yield to maturity rates on government bonds). To detect and analyze the anchoring bias, we adopt the experimental design consistent with Jacowitz and Kahneman (1995). We randomly attribute our participants to one of the two groups: (i) "Control" group (Group C): participants in this group are given no additional information and asked to provide their best estimate for each of the 
questions, and (ii) "Anchoring" group (Group A): participants in this group are asked the same questions, yet, before each question they receive unrelated economic or financial indicators ("anchor indicators"), of the same order of magnitude and expressed at the same scale. Since anchoring bias is supposed "to draw people's estimates closer to the anchor", we expect that the answers given by participants in Group A should be closer to anchor indicators than the answers given by participants in Group C, and therefore, in order to measure the degree of anchoring bias, for each person and for each question, we compare the deviations of both group's answers from the anchor indicators.

We document that significant anchoring bias is exhibited, on average, for each of our experimental questions and by the majority of the participants in Group $A$, the degree of the bias being higher for women and older participants. Furthermore, as a major hypothesis of our research, we expect the anchoring bias to be more strongly pronounced in cases where the extent of participants' pre-existing knowledge is narrower. Respectively, we divide our experimental questions into two categories, according to the difficulty of recalling the answers, and analyze the differences in anchoring measures between the categories of questions. We find that people exhibit significantly stronger anchoring bias for "difficult" than for "easy" questions. The difference persists for all groups of participants in our sample.

Our findings indicate that the less people know about an object or a category, the more they may be influenced by some, even arbitrary, anchors when estimating its characteristics or forecasting related future outcomes, and consequently, the wider the field for potential manipulations from the viewpoint of those who might be interested, for example, in convincing people to invest into a stock or to buy a product.

The rest of the paper is structured as follows. In Section 2, we review the literature on anchoring, featuring both psychological aspects and economic applications. In Section 3, we describe our experimental design and research approach. Section 4 defines our hypotheses and provides the empirical tests and the results. Section 5 concludes and provides a brief discussion.

\section{LITERATURE REVIEW}

Psychological evidence and implications of anchoring: Human judgments fall prey to a variety of systematic biases and distortions (for an overview, see, for example, Kahneman, et al. (1982)). Tversky and Kahneman (1974) propose that in assessing the likelihood of uncertain events and predicting or recalling certain values or outcomes, people rely on a number of simplifying rules of decision-making, called heuristics. One of the heuristics they discuss is the process of anchoring (or anchoring bias). They argue that in many situations people make estimates by considering an initial value that they adjust upwards or downwards to yield a final estimate. Such adjustments are often insufficient, leaving judgments biased in the direction of the initial "anchor" value. In what is probably the best-known demonstration of this effect, Tversky and Kahneman (1974) first ask their research participants whether the percentage of African nations in the United Nations (target number) is higher or lower than an arbitrary number (the anchor) which is randomly determined by spinning a wheel of fortune (e.g., $65 \%$ or $10 \%$ ). Participants are then asked to give their best estimate of this percentage. Absolute judgments are assimilated to the provided anchor value so that the mean estimate of participants who received the high anchor was $45 \%$, compared to $25 \%$ for participants who received the low anchor.

Anchoring effects have proved to be a truly ubiquitous phenomenon that has been observed in a broad array of different judgmental domains (for review, see, for example, Mussweiler and Strack (1999a), English (2008)). Jacowitz and Kahneman (1995) ask students a number of general knowledge questions (like length of Mississippi or height of Everest), and report that participants who are given high anchors provide higher estimates than those who are given low anchors. Similar results are also obtained by Strack and Mussweiler (1997) and Mussweiler and Strack (1999b).

Cervone and Peake (1986) document that people receiving high anchors subsequently estimate their own capabilities higher than those who are given low anchors. Plous (1989) argues that the anchoring bias affects people's probability assessments. Furthermore, Chapman and Johnson (1994) ask people to evaluate a number of lotteries varying in their expected values and ranges, and find that the higher the anchor they are given, the higher the minimal sum for which they would sell the lottery.

Anchoring effects are well-documented in legal judgment. Markovsky (1988) reports that subjects exposed to large monetary anchors suggest higher rewards for a witness who comes forward to testify about a crime. Chapman and Bornstein (1996) ask 
their experiment participants to act as jurors and to decide on the amount of personal injury compensation for a specific case, and find that the higher the requested compensation, serving as a random anchor, the higher the compensation actually awarded by the "jurors". This may constitute a really interesting result, implying that the more people ask for, the more they get. In the same spirit, English and Mussweiler (2001) carry an experiment involving a group of professional judges, and conclude that sentencing decisions are anchored towards the sentences demanded by prosecutors. The magnitude of this influence proves to be dramatic, as judges who consider a high demand of 34 months give final sentences (for the same crimes) that are almost 8 months longer than judges who consider low demand of 12 months. The effect appears to be independent of judges' experience.

A large number of studies consider the effects of anchoring in a variety of additional domains. Ehrbeck and Waldman (1996) concentrate on the existing evidence that professional forecasters in various domains make predictable forecast errors persisting over time, and construct a formal behavioral model implying that making repeated forecasts, the forecasters may be anchored towards their own previous forecasts and the prediction patterns typical of able forecasters. English (2008) asks a group of students to estimate the average price of a German midsize car, after providing them both a standard anchoring and some additional, relevant or irrelevant, information, and finds that the estimates are biased towards the anchor and that relevant knowledge decreases the effect of anchoring. Bowman and Bastedo (2010) analyze the anchoring effects in assessments of institutional reputation, and document that world university rankings published by Times Higher Education Supplement influence peer assessments of reputation in subsequent surveys.

Not only is the anchoring effect influential in a plethora of laboratory and real-world settings, this influence is also remarkably robust. In particular, anchoring is independent of many potentially moderating variables. For one thing, anchoring occurs even if the anchor values are clearly uninformative for the critical estimate, for example, because they were randomly selected (Mussweiler and Strack (2000), Tversky and Kahneman (1974)). Moreover, anchoring remains uninfluenced by the extremity of the anchor (Chapman and Johnson (1994), Strack and Mussweiler (1997)) so that even implausibly extreme values yield an effect. For example, in the study by Strack and Mussweiler (1997), estimates for Mahatma Gandhi's age are assimilated to an unreasonably high anchor value of 140 years. Furthermore, anchoring effects appear to be independent of participants' motivation (Wilson, et al. (1996)). Specifically, the attempts to improve accuracy by awarding a prize for the best estimate prove unsuccessful. In addition, it has been demonstrated that anchoring occurs independently of participants' expertise (Englich and Mussweiler (2001)). Furthermore, anchoring effects are characterized by an exceptional temporal robustness and persist over fairly long periods of time. For example, in a study by Mussweiler (2001), anchoring effects are still apparent a week after the anchor value had been considered. Probably the most striking demonstration of the robustness of the phenomenon, however, stems from research demonstrating that explicit instructions to correct for a potential influence of an anchor do not mitigate the effect (Wilson et al. (1996)). Even explicitly forewarning judges about the potential distortion and informing them about its direction does not diminish the effect. This suggests that anchoring is an exceptionally robust phenomenon that is difficult to avoid.

Economic applications of anchoring: The vast research on anchoring originates from psychology, and takes roots in a number of fields and domains. Still, by the present moment, the applications of the effect of anchoring that may be classified as "economic" are relatively scarce.

Northcraft and Neale (1987) demonstrate that realestate pricing decisions depend on the listing price for the property. They have real-estate agents and non-professionals estimate the value of a property. Participants are given a ten-page booklet including all the information that is important for real-estate pricing and the listing price of the house, either above or below the actual appraisal value of the property. Replicating the typical anchoring finding, the authors document that participants' estimates for the value of the property are assimilated towards the provided anchors. Similar results are obtained both for experts and amateur subjects.

Gruen and Gizycki (1993) use anchoring to explain the widely-observed anomaly that forward discounts do not properly explain subsequent exchange rate movements. The anchoring phenomenon may be relevant to the "sticky prices" that are so talked about by macroeconomists. So long as past prices are taken as suggestions of new prices, the new prices 
will tend to be close to the past prices. The more ambiguous the value of a commodity, the more important a suggestion is likely to be, and the more important anchoring is likely to be for price determination.

Fisher and Statman (2000) discuss economic implications of a number of behavioral biases and suggest that stock market analysts may employ mean historical dividend yields and price-earnings ratios as anchors for forecasts of future dividend yields and price-earnings ratios, though historical figures diverge from their means by wide margins, and so may the future figures.

Galinsky and Mussweiler (2001) explore the role of anchoring in buyers' and sellers' behavior and their subsequent profits. They show that first offers may influence the final negotiation outcomes, because they serve as judgmental anchors to which the final outcomes are assimilated. They also demonstrate that whichever party, the buyer or the seller, makes the first offer obtains a better outcome from her viewpoint. Zielonka (2004) carries an experiment involving financial analysts, and finds that certain historical peaks and lows in security and index quotes serve as mental anchors in technical analysis.

The effect of anchoring is also analyzed in the context of consumer decision-making. Biswas and Burton (1993) suggest that price claims in advertisements influence consumer behavior, because they function as anchors in product evaluation. Simonson and Drolet (2004) report the effect of anchoring on consumers' willingness-to-pay and willingness-to-accept.

Beggs and Graddy (2009) document anchoring effect in art auctions by showing that art works may be sold at much higher prices in "hot" markets when the auction buyers may be anchored by high prices that were previously set.

Overall, previous economic studies dealing with anchoring concentrate on documenting the bias itself, and on its potential implications. To our best knowledge, there are yet no studies analyzing the effect of pre-existing people's knowledge on the degree of exhibited anchoring bias, in economic settings. Present study makes an effort to fill this gap.

Experimental design and research approach: Previous studies documenting the anchoring effects employ two major approaches of introducing an anchor:
1. Standard anchoring: According to this approach, people are exposed to an external anchor, and then sequentially make a comparative and an absolute judgment about the critical target quantity (for example, Tversky and Kahneman (1974), Chapman and Johnson (1994)).

2. Basic anchoring: This approach does not involve a direct comparison of anchor and target. Here, merely increasing the accessibility of the anchor value prior to the critical estimate is sufficient to produce anchoring effects. For example, Wilson et al. (1996) ask students to copy either five pages of high numbers or five pages of words before estimating the number of fellow students who would get cancer. Those who have copied five pages of high numbers appear to give higher estimates than those who have copied five pages of words. Many other papers (for example, Northcraft and Neale (1987), English and Mussweiler (2001)) explicitly provide some "starting points" (listing price, sentence demanded, etc.), and then ask for the target estimates, without comparing them to the anchors.

In present study, we adopt the second approach. We run an experiment involving 67 MBA students from the Technion, Israel Institute of Technology, and the University of Haifa ${ }^{1}$. We ask our participants to recall a number of recent economic and financial indicators for Israeli economy, in general, and Tel Aviv Stock Exchange, in particular. For this kind of questions, MBA students may serve a competent audience. To introduce and to control for the effect of anchoring, we randomly attribute our participants to one of the two groups ${ }^{2}$ :

- "Control" group (Group C): Participants in this group are given no additional information and asked to provide their best estimate for each of the respective indicators.

- $\quad$ "Anchoring" group (Group A): Participants in this group are asked the same questions,

${ }^{1} 40$ males and 27 females with mean age of 33.5 took part in the experiment, 26 of them at the Technion and 41 at the University of Haifa.

${ }^{2}$ There were 32 participants in Group C (21 males and 11 females, with mean age of 33.3), and 35 participants in Group A (19 males and 16 females, with mean age of 34.2). 
yet, before each question they receive unrelated economic or financial indicators ("anchor indicators"), of the same order of magnitude and expressed at the same scale. For example, we provide the current value of S\&P 500 Index and ask the participants to provide their best estimate for the current value of TA-25 Index ${ }^{3}$ - clearly, unrelated figure.

In Appendix B, we present the experimental questionnaire for Group $A$, as provided to the participants at the Technion ${ }^{4}$.

Since anchoring bias is supposed "to draw people's estimates closer to the anchor", the general intuition says that the answers given by participants in Group A should be closer to anchor indicators than the answers given by participants in Group $C^{5}$.

To calculate the measures of anchoring, we use the method, which is consistent with that proposed by Jacowitz and Kahneman (1995). First of all, we calculate the anchoring measure for each answer given by each participant in group $A$ in the following way:

$$
A_{n}^{i}=1-\frac{\left\|R A_{n}^{i}-I_{n}\right\|}{D C_{n}}(1)
$$

where: $A_{n}^{i}$ - Anchoring (bias) measure for question $n$ and person $i, R A_{n}^{i}$ - actual answer (Response) to question $n$ given by participant $i$ from Croup A, $I_{n}$ -

${ }^{3}$ Index that tracks the prices of the shares of the 25 companies with the highest market capitalization on the Tel Aviv Stock Exchange.

4 The experiment was run on May 13, 2010 at the Technion, and on May 21, 2010 at the University of Haifa. Since a part of the "anchor indicators" (questions 1, 4, 9, $18,20)$ are updated on daily basis, they were different for Group $A$ at the Technion and at the University of Haifa. Group C questionnaires, of course, included the same questions without "anchor indicators". In all questionnaires, we asked for participants' personal details (sex, age, previous education).

${ }^{5}$ A number of previous studies provide different anchors to two different groups, and subsequently suggest that each group's answers should be closer to the respective anchor. We use the Control group and let the answers of its participants be "independent" of any anchors, and therefore, suggest that the answers in the Anchoring group should be closer to the anchor. anchor Indicator (anchor) for question $n, D C_{n}$ - mean Deviation from the anchor for question $n$, in Group C, which, in its turn, is calculated as:

$$
D C_{n}=\frac{\sum_{j=1}^{N C}\left\|R C_{n}^{j}-I_{n}\right\|}{N C}
$$

where: $R C_{n}^{j}$ - actual answer (Response) to question $n$ given by participant $j$ from Group C, NC - Number of participants in group C (32 participants).

Participant $i$ from Group A who does not exhibit anchoring bias should provide the same value of $R A_{n}^{i}$ that she would have provided without being exposed to the anchor, i.e. probably, the same value as her $R C_{n}^{i}$ would have been if she were by herself a part of Group C. That is, in terms of Equations 1 and 2 , without anchoring bias, the deviations of $R A_{n}^{i}$ and $R C_{n}^{i}$ from $I_{n}$ should not be different ${ }^{6}$, and therefore, the value of $A_{n}^{i}$ should be equal to zero. The maximal value of the anchoring measure is equal to one and is obtained for a participant in Group A that, having received an anchor, provides the answer to question $n$ that is exactly equal to the anchor. $A_{n}^{i}$ may get any value. For example, it may be negative if participant $i$ from Group A provides an answer to question $n$ that deviates from the anchor farther than do, on average, the answers in Group C.

As follows from Equations 1 and 2, we employ absolute individual, rather than average, deviations of actual answers from the anchors. This approach arises from the fact that both $R A_{n}^{i}$ and $R C_{n}^{j}$ may be on average equal, and in this case, the use of averages may produce meaningless results. In contrast, employing the absolute individual deviations allows us to detect, for each participant in Group A, if her (anchor-affected) answer is closer to the anchor than it would probably have been without it. In other words, in order to arrive at mean anchoring measures, one should start from the individual measures, and that is what we do.

${ }^{6}$ On average, given that Groups $\mathrm{C}$ and $\mathrm{A}$ are similar by their participants' characteristics, which is ensured by randomly attributing students from the same class to one of the two groups. 
Furthermore, we do calculate the mean anchoring measures:

- for each question:

$$
A Q_{n}=\frac{\sum_{i=1}^{N H} A_{i}^{n}}{N A}
$$

where: $A Q_{n}$ - mean Anchoring measure for Question $n, N A$ - Number of participants in group $A$ (35 participants).

and

- for each participant $i$ in Group A:

$$
A P^{i}=\frac{\sum_{n=1}^{N Q} A_{i}^{n}}{N Q}
$$

where: $A P^{i}$ - Personal Anchoring measure for participant $i, N Q$ - Number of Questions in the questionnaire (21 questions).

Testable hypotheses and results: The major goal of our paper is to analyze the effect of pre-existing people's knowledge on the degree of anchoring bias they exhibit. In this context, we first of all calculate mean anchoring measures for each of the questions and for each of the participants, and then analyze the differences in these measures for different groups of questions.

Anchoring bias, by experimental questions: At the first stage, we calculate the anchoring measures $\left(A_{n}^{i}\right)$ for each question and for each participant, and subsequently, the mean (over 35 participants from Group A) anchoring measures $\left(A Q_{n}\right)$ for each of the experimental questions. We expect that participants in Group A will, on average, provide answers that will be closer to the respective anchor indicators than those by participants in Group C. Therefore, for each of the 21 experimental questions, we hypothesize the following:

Hypothesis 1:

HO: $A Q_{n}=0$ (no anchoring bias for question $\left.n\right)$

H1: $A Q_{n}>0$ (positive anchoring bias for question $n$ )
Table 1 comprises summary statistics of $A_{n}^{i}$ separately for each of the experimental questions, and the tests of Hypothesis 1. The results strongly indicate the existence of anchoring bias for all the questions. All the 21 mean anchoring measures are positive, majority of them significantly (18 at $5 \%$ level, including 16 at $1 \%$ level), ranging from 0.042 to 0.744 . Moreover, for all the questions, vast majority of the participants (for one question, even all of them) exhibit the bias.

Anchoring bias, by participants and categories of participants: Having documented the anchoring effect for each of the questions, we henceforth calculate the mean (over 21 questions) personal anchoring measures $\left(A P^{i}\right)$ for each of the participants in Group A. Furthermore, we analyze these personal anchoring measures for the total sample and for the subsamples by the following classifications:

a) Sex: males (19 participants) and females (16 participants).

b) Age: we divide our sample in two roughly equal age categories: (i) younger than 33 (17 participants), and (ii) 33 and older (18 participants).

c) Previous education: B.A./B.Sc. (23 participants) and M.A./M.Sc. (12 participants) academic degree.

In this personal context, we expect that the participants in Group A exhibit the anchoring bias, and therefore, for the total sample and for each of the subsamples, we hypothesize as follows:

Hypothesis 2:

HO: Mean $A P^{i}=0$ (no anchoring bias for the respective sample/subsample)

H1: Mean $A P^{i}>0$ (positive anchoring bias for the respective sample/subsample)

Table 2 presents summary statistics of $A P^{i}$ for the total sample and for each of the subsamples, and the tests of Hypothesis 2. The results indisputably support the latter. On average, 33 out of 35 participants in our experiment exhibit anchoring bias, their personal anchoring measures ranging from -0.178 to 0.830 (with the general mean of 0.450 ). Clearly, the means of the personal measures are significantly positive for all the categories of the participants. In addition, we observe that the anchoring bias is stronger pronounced for females 
and for older participants, while previous education seems to have little effect on the degree of the bias.

Effect of the previous knowledge on the degree of the anchoring bias: In this Subsection, we make an effort to shed light on the effect of people's knowledge on a specific matter on the degree of anchoring bias they exhibit.

For this purpose, we need to have some idea about the judges' knowledge. Mussweiler and Strack (2000) argue that this is quite a difficult task, since judges' knowledge about the target objects may vary substantially. Therefore, they manipulate their participants' knowledge by increasing the availability of a specific category before asking questions about the target. They conclude that the less people know about the target object, the more they assimilate their estimates to the anchor. In the same spirit, English (2008) provides experiment participants with either relevant of irrelevant information, and finds that relevant knowledge decreases the magnitude of basic anchoring effect.

In this study, we concentrate on the effect of preexisting knowledge on the magnitude of anchoring bias. Unlike the above-mentioned studies, we do not manipulate our participants' knowledge, but rather build our experimental questionnaire so as to allow us classifying the questions by the difficulty of answering them, or in other words, by the amount of pre-existing knowledge people have on them. We ask similar recall questions for the more and less recent periods, that are respectively less and more difficult to recall. For example, Question 2 requires the participants to recall the annual return on TA-25 Index in 2009, while Question 3 refers to the average annual return on TA-25 Index over years 2007-2009, and Question 4 asks for the distance of the current value of TA-25 Index from the Index's historical high. So, we may expect that our participants, being rather competent in stock market matters, should find it easier to answer Question 2 than Questions 3 and 4, and respectively, should exhibit stronger anchoring bias for Questions 3 and 4. In such a way, we classify our experimental questions as:

1. Questions that are relatively more easy to answer ("easy") - questions referring to the present moment or to relatively more recent periods (2009 versus 2007-2009, or first five months of 2010 versus 2009).

2. Questions that are relatively more difficult to answer ("difficult") - questions referring to less recent periods, and questions on the current yield-to-maturity rates on two kinds of mid-term government bonds (Questions 11 and 12 , requiring more extensive knowledge of capital markets).

Overall, our questionnaire includes 11 "easy" questions (Questions 1, 2, 5, 8, 10, 13, 14, 16, 17, $18,20)$ and 10 "difficult" questions (Questions 3, 4, 6, $7,9,11,12,15,19,21)$.

Consequently, according to the intuition above, we expect the anchoring bias to be more pronounced for the "difficult" than for the "easy" questions, or in other words, we suggest that mean anchoring measures $\left(A Q_{n}\right)$ will, on average, be higher for the "difficult" than for the "easy" questions. Respectively, for both groups of questions, we calculate the means and the medians of $A Q_{n}$, and hypothesize the following:

Hypothesis 3:

$\mathrm{HO}$ :

Mean / Median $A Q_{n}("$ difficult") = = Mean / Median $A Q_{n}$ ("easy") (similar degree

of anchoring bias for both groups of questions)

H1:

Mean / Median $A Q_{n}("$ difficult") > $>$ Mean/Median $A Q_{n}$ ("easy") (stronger anchoring bias for "difficult" than for "easy" questions)

Table 3 reports summary statistics of $A Q_{n}$ for the total sample and separately for both categories of questions, and test statistics of Hypothesis 3. First of all, as we have already noted in Subsection 4.1, mean anchoring measures are positive for all the questions in our questionnaire ${ }^{7}$. Furthermore, we observe that, consistently with Hypothesis 3, the mean and the median of $A Q_{n}$ are significantly higher for the "difficult" (0.569 and 0.570 , respectively) than for the "easy" (0.341 and 0.378 , respectively) questions. That is, our participants exhibit stronger anchoring bias answering more difficult questions. In other words, the more difficult is

${ }^{7}$ We may also mention that the mean $A Q_{n}$ for the total sample (21 questions) is exactly equal to the mean personal anchoring measure for the total sample (35 participants) reported in Table 2 ( 0.450 both). Of course, the "order of calculation of means" (first, over the participants, and then, over the questions, or vice versa) does not change the final result. 
to recall an answer, the more it is affected by any kinds of anchors being provided.

Now, in order to provide additional support for our findings, we perform another test aimed at comparing the degree of anchoring bias for the "easy" and the "difficult" questions. For each of the participants in Group A, we calculate mean personal anchoring measures separately for both categories of questions:

$$
A P E^{i}=\frac{\sum A_{i}^{n} \mid \text { easy }}{11}(5)
$$

and: $\quad A P D^{i}=\frac{\sum A_{i}^{n} \mid \text { difficult }}{10}$ (6)

where: $A P E^{i}$ - mean Personal Anchoring measure for "Easy" questions for participant $i, A P D^{i}$ - mean Personal Anchoring measure for "Difficult" questions for participant $i, \sum A_{i}^{n} \mid$ easy - sum of participant i's Anchoring measures for "easy" questions, $\sum A_{i}^{n} \mid$ difficult - sum of participant i's Anchoring measures for "difficult" questions.

Consequently, we expect that, on average, mean personal anchoring measures for the "difficult" questions should be higher than for the "easy" ones. That is, for the total sample and for each one of the subsamples, as defined above, we hypothesize that:

Hypothesis 4:

HO:

Mean / Median $A P D^{i}=$ Mean / Median $A P E^{i}$ (si milar degree of anchoring bias for both groups of questions)

$$
\text { H1: }
$$

Mean/Median $A P D^{i}>$ Mean/Median $A P E^{i}$ (stronger anchoring bias for "difficult" than for "easy" questions)

Table 4 presents summary statistics of $A P E^{i}$ and $A P D^{i}$ for the total sample and for each of the subsamples, and the tests of Hypothesis 4, which is strongly supported by the results. Mostly important the mean and the median of $A P D^{i}$ for the total sample (0.569 and 0.617 , respectively) are significantly higher than the mean and the median of
$A P E^{i}$ (0.341 and 0.451 , respectively). This relation also holds for all the subsamples, the differences being more pronounced for men, younger, and less educated participants. Thus, we may conclude that on the "individual level", our participants exhibit stronger anchoring bias for relatively more difficult questions.

Overall, the results in this Subsection, probably making up the major contribution of our paper, demonstrate that the more difficult is a question, the stronger is the influence of any arbitrary anchor on the answer provided. This result is consistent with Mussweiler and Strack (2000) and English (2008), and also with the findings of the recent research by Kudryavtsev and Cohen (2010), showing that people exhibit stronger "hindsight bias" (tendency to overestimate ex-post, or in hindsight, how predictable an outcome was in foresight ${ }^{8}$ ) for the questions that are more difficult to answer. Moreover, our result may be, in fact, seen in line with conclusions of a number of empirical studies in behavioral finance showing that, generally, the less people know about a matter, the more they are inclined to resort to various kinds of simplifying decision-making rules. For example, Baker and Wurgler (2006) argue that stocks of low capitalization, growth, and highly volatile stocks (which market investors probably have less information about) are especially likely to be disproportionately sensitive to broad waves of investor sentiment. Similarly, Kliger and Kudryavtsev (2010) demonstrate that investors trading in small and more volatile stocks are more affected by the availability heuristic (people's tendency to determine the likelihood of an event according to the easiness of recalling similar instances, and, thus, to overweight current information, as opposed to processing all relevant information ${ }^{9}$ ).

\section{CONCLUSIONS AND DISCUSSION:}

Our paper explores the role of anchoring in perceiving economic and financial information, and, in particular, the effect of pre-existing knowledge on the degree of this bias.

Employing an extensive experimental questionnaire and an audience which is sufficiently competent in

\footnotetext{
${ }^{8}$ See, for example, studies by Pohl (2004), Blank et al. (2007) and (2008), Erdfelder et al. (2007) for the explanation and the discussion on the hindsight bias.

9 See Tversky and Kahneman (1973, 1974) for the explanation and the discussion on the availability heuristic.
} 
economic and financial matters, we reveal the effect of anchoring in recalling real-world outcomes. We find that the effect is exhibited, on average, for each of our experimental questions and by vast majority of the participants, its degree being higher for women and older participants.

Furthermore, we hypothesize that anchoring bias is more strongly pronounced for the questions that are more difficult to answer. We divide our experimental questions into two categories, according to the difficulty of recalling the answers, and analyze the differences in anchoring measures between the categories of questions. The results support our hypothesis, indicating that our participants exhibit significantly stronger anchoring bias for "difficult" than for "easy" questions. The difference persists for all groups of participants in our sample.

Our findings may have important implications for decision-making in various fields of real life. We not only document that decision-making may be influenced by some arbitrary anchors, but find that the magnitude of this influence is the more pronounced, the less people know about the target. That is, for example, when an investor considers purchasing a stock and respectively, tries to forecast its future returns, the less fundamental information she has about the issuing company, the more she will be affected by any anchors to which she is currently exposed.

Both "sides of the game" might pay attention to this result. On the one hand, everyone who is willing to sell an asset and has some potential of manipulating the buyers' knowledge about the asset (stock issuers, product manufacturers) may consider providing relatively little relevant information about the asset, but instead integrating some, not really relevant, high figures in the product description, in order to increase the buyers' estimates for the product price. On the other hand, potential buyers should look for the fundamentals and think twice when estimating how much a relatively new and unknown product is worth for them.

\section{REFERENCES}

Baker, M., and J. Wurgler, 2006, Investor Sentiment and the Cross-Section of Stock Returns, Journal of Finance, 61, 1645-1680.

Beggs, A., and K. Graddy, 2009, Anchoring Effects: Evidence from Art Auctions, American Economic Review, 99, 1027-1039.
Biswas, A., and S. Burton, 1993, Consumer Perceptions of Tensile Price Claims in Advertisements: An Assessment of Claim Types across Different Discount Levels, Journal of the Academy of Marketing Science, 21, 217-229.

Blank, H., J. Musch, and R. F. Pohl, 2007, Hindsight Bias: On Being Wise after the Event, Social Cognition, 25, 19.

Blank, H., G. von Collani, V. Fischer, and S. Nestler, 2008, How Many Hindsight Biases are There? Cognition, 106, 1408-1440.

Bowman, N. A., and M. N. Bastedo, 2010, Anchoring Effects in World University Rankings: Exploring Biases in Reputation Scores, Higher Education, Forthcoming.

Cervone, D., and P. K. Peake, 1986, Anchoring, Efficacy, and Action: The Influence of Judgmental Heuristics on Self-Efficacy Judgment and Behavior, Journal of Personality and Social Psychology, 50, 492-501.

Chapman, G. B., and B. H. Bornstein, 1996, The More You Ask for, the More You Get: Anchoring in Personal Injury Verdicts, Applied Cognitive Psychology, 10, 519-540.

Chapman, G. B., and E. J. Johnson, 1994, The Limits of Anchoring, Journal of Behavioral Decision Making, 7, 223-242.

English, B., 2008, When Knowledge Matters-Differential Effects of Available Knowledge in Standard and Basic Anchoring Tasks, European Journal of Social Psychology, 38, 896-904.

English, B., and T. Mussweiler, 2001, Sentencing under Uncertainty: Anchoring Effects in the Courtroom, Journal of Applied Social Psychology, 31, 1535-1551.

Ehrbeck, T., and R. Waldman, 1996, Why are Professional Forecasters Biased? Agency Versus Behavioral Explanations, Quarterly Journal of Economics, 111, 21-40.

Erdfelder, E., M. Brandt, and A. Broder, 2007, Recollection Biases in Hindsight Judgments, Social Cognition, 25(1), 114-131.

Fisher, K. L., and M. Statman, 2000, Cognitive Biases in Market Forecasts, Journal of Portfolio Management, 27, 72-81.

Galinsky, A. D., and T. Mussweiler, 2001, First Offers as Anchors: The Role of Perspective-Taking and Negotiator Focus, Journal of Personality and Social Psychology, 81, 657-669.

Gruen, D. K., and M. C. Gizycki, 1993, Explaining Forward Discount Bias: Is It Anchoring? Princeton University Woodrow Wilson School Discussion Paper in Economics, 164. 
Jacowitz, K. E., and D. Kahneman, 1995, Measures of Anchoring in Estimation Tasks, Personality and Social Psychology Bulletin, 21, 1161-1166.

Kahneman, D., P. Slovic, and A. Tversky, 1982, Judgment under Uncertainty: Heuristics and Biases, New York: Cambridge University Press.

Kliger, D., and A. Kudryavtsev, 2010, The Availability Heuristic and Investors' Reaction to Company-Specific Events, Journal of Behavioral Finance, 11 (1), 50-65.

Kudryavtsev, A., and G. Cohen, 2010, The Less I Remember, the More Confident I Am: Hindsight Bias in Economic and Financial Knowledge, Working Paper, Academic College of Emek Yezreel.

Markovsky, B, 1988, Anchoring Justice, Social Psychology Quarterly, 51, 213-224.

Mussweiler, T, 2001, The Durability of Anchoring Effects, European Journal of Social Psychology, 31, 431-442.

Mussweiler, T., and F. Strack, 1999a, Comparing is Believing: A Selective Accessibility Model of Judgmental Anchoring, In W. Stroebe \& M. Hewstone (Eds.), European review of social psychology (Vol. 10, pp. 135-167). Chichester, UK: Wiley.

Mussweiler, T., and F. Strack, 1999b, HypothesisConsistent Testing and Semantic Priming in the Anchoring Paradigm: A Selective Accessibility Model, Journal of Experimental Social Psychology, 35, 136164.

Mussweiler, T., and F. Strack, 2000, Numeric Judgment under Uncertainty: The Role of Knowledge in Anchoring, Journal of Experimental Social Psychology, 36, 495-518.

Northcraft, G. B., and M. A. Neale, 1987, Experts, Amateurs, and Real Estate: An Anchoring-and-
Adjustment Perspective on Property Pricing Decisions, Organizational Behavior and Human Decision Processes, 39, 84-97.

Pohl, R.F., 2004, Hindsight Bias, In R.F. Pohl (Ed.), Cognitive Illusions: A Handbook on Fallacies and Biases in Thinking, Judgment and Memory, Hove, UK: Psychology Press, 363-378.

Plous, S, 1989, Thinking the Unthinkable: The Effects of Anchoring on Likelihood Estimates of Nuclear War, Journal of Applied Social Psychology, 19, 67-91.

Simonson, I., and A. Drolet, 2004, Anchoring Effects on Consumers' Willingness-to-Pay and Willingness-toAccept, Journal of Consumer Research, 31, 681-690.

Strack, F., and T. Mussweiler, 1997, Explaining the Enigmatic Anchoring Effect: Mechanisms of Selective Accessibility, Journal of Personality and Social Psychology, 73, 437-446.

Tversky, A., and D. Kahneman, 1973, Availability: A Heuristic for Judging Frequency and Probability, Cognitive Psychology, 4, 207-232.

Tversky, A., and D. Kahneman, 1974, Judgment under Uncertainty: Heuristics and Biases, Science, 185, 1124-1131.

Wilson, T. D., C. Houston, K. M. Etling, and N. Brekke, 1996, A New Look at Anchoring Effects: Basic Anchoring and its Antecedents, Journal of Experimental Psychology: General, 4, 387-402.

Zielonka, P., 2004, Technical Analysis as the Representation of Typical Cognitive Biases, International Review of Financial Analysis, 13, 217225 


\section{Appendix A: Tables}

Table 1

Anchoring measure statistics, by questions

The table reports, by questions, summary statistics of the anchoring measures $\left(A_{n}^{i}\right)$ calculated for each of the participants in Group A as follows:

$A_{n}^{i}=1-\frac{\left\|R A_{n}^{i}-I_{n}\right\|}{D C_{n}}$ with $D C_{n}=\frac{\sum_{j=1}^{N C}\left\|R C_{n}^{j}-I_{n}\right\|}{N C}$

Where: $A_{n}^{i}$ - Anchoring (bias) measure for question $n$ and person $i, R A_{n}^{i}$ - actual answer (Response) to question $n$ given by participant $i$ from Croup A, $I_{n}$ - anchor Indicator (anchor) for question $n, D C_{n}$ - mean Deviation from the anchor for question $n$, in Group $C, R C_{n}^{j}$ - actual answer (Response) to question $n$ given by participant $j$ from Group C, NC - Number of participants in group C (32 participants).

The last column presents, for each question, the statistics for the hypothesis that the mean anchoring measure is greater than zero, which is consistent with Group A participants on average exhibiting anchoring bias, for the respective question.

\begin{tabular}{|c|c|c|c|c|c|c|c|}
\hline \multirow[t]{2}{*}{ Question No. } & \multicolumn{6}{|c|}{ Anchoring measure $\left(A_{n}^{i}\right)$} & \multirow{2}{*}{$\begin{array}{c}A Q_{n}>0: \\
\text { t-statistic } \\
(p-\text {-value, } \%)\end{array}$} \\
\hline & $\begin{array}{l}\text { Mean } \\
\left(A Q_{n}\right)\end{array}$ & Median & $\begin{array}{l}\text { Standard } \\
\text { Deviation }\end{array}$ & Maximum & Minimum & $\begin{array}{l}\text { No. (percent) } \\
\text { of positive }\end{array}$ & \\
\hline 1 & 0.641 & 0.678 & 0.353 & 0.971 & -0.465 & 34 (97.14) & $10.75(0.00)$ \\
\hline 2 & 0.396 & 0.675 & 0.912 & 0.978 & -2.800 & 31 (88.57) & $2.57(1.48)$ \\
\hline 3 & 0.704 & 0.843 & 0.370 & 0.980 & -0.343 & 32 (91.43) & $11.26(0.00)$ \\
\hline 4 & 0.718 & 0.855 & 0.300 & 0.991 & -0.252 & 34 (97.14) & $14.17(0.00)$ \\
\hline 5 & 0.042 & 0.348 & 1.224 & 0.980 & -3.788 & 26 (74.29) & $0.20(83.92)$ \\
\hline 6 & 0.421 & 0.654 & 0.552 & 0.994 & -0.868 & 29 (82.86) & $4.51(0.01)$ \\
\hline 7 & 0.422 & 0.696 & 0.636 & 0.993 & -1.382 & 25 (71.43) & $3.92(0.04)$ \\
\hline 8 & 0.173 & 0.654 & 1.141 & 0.992 & -3.850 & 27 (77.14) & $0.90(37.52)$ \\
\hline 9 & 0.744 & 0.729 & 0.212 & 0.995 & 0.174 & 35 (100.00) & $20.72(0.00)$ \\
\hline 10 & 0.378 & 0.596 & 0.592 & 0.954 & -1.613 & $26(74.29)$ & $3.78(0.06)$ \\
\hline 11 & 0.396 & 0.425 & 0.419 & 0.934 & -0.308 & 25 (71.43) & $5.60(0.00)$ \\
\hline 12 & 0.440 & 0.496 & 0.456 & 0.976 & -0.416 & 27 (77.14) & $5.70(0.00)$ \\
\hline 13 & 0.292 & 0.665 & 0.656 & 0.888 & -1.851 & 25 (71.43) & $2.64(1.25)$ \\
\hline 14 & 0.544 & 0.737 & 0.576 & 0.934 & -2.480 & 34 (97.14) & $5.58(0.00)$ \\
\hline 15 & 0.701 & 0.828 & 0.259 & 0.994 & -0.015 & 34 (97.14) & $15.99(0.00)$ \\
\hline 16 & 0.276 & 0.270 & 0.561 & 0.948 & -1.919 & 27 (77.14) & $2.92(0.62)$ \\
\hline 17 & 0.044 & 0.091 & 0.942 & 0.921 & -4.215 & 19 (54.29) & $0.28(78.39)$ \\
\hline 18 & 0.573 & 0.832 & 0.609 & 0.993 & -1.961 & $29(82.86)$ & $5.57(0.00)$ \\
\hline 19 & 0.712 & 0.836 & 0.322 & 0.983 & -0.224 & 33 (94.29) & $13.09(0.00)$ \\
\hline 20 & 0.389 & 0.384 & 0.459 & 0.997 & -0.982 & 30 (85.71) & $5.01(0.00)$ \\
\hline 21 & 0.434 & 0.640 & 0.391 & 0.924 & -0.406 & $28(80.00)$ & $6.58(0.00)$ \\
\hline
\end{tabular}


Table 2

Personal anchoring measure statistics, by categories of participants

The table reports, by categories of participants, summary statistics of the personal anchoring measures $\left(A P^{i}\right)$ calculated for each of the participants in Group A as follows:

$A P^{i}=\frac{\sum_{n=1}^{N Q} A_{i}^{n}}{N Q}$ with $A_{n}^{i}=1-\frac{\left\|R A_{n}^{i}-I_{n}\right\|}{D C_{n}}$ and $D C_{n}=\frac{\sum_{j=1}^{N C}\left\|R C_{n}^{j}-I_{n}\right\|}{N C}$

Where: $A P^{i}$ - Personal Anchoring measure for participant $i, N Q$ - Number of Questions in the questionnaire (21 questions), $A_{n}^{i}$ - Anchoring (bias) measure for question $n$ and person $i, R A_{n}^{i}$ - actual answer (Response) to question $n$ given by participant $i$ from Croup A, $I_{n}$ - anchor Indicator (anchor) for question $n, D C_{n}-$ mean Deviation from the anchor for question $n$, in Group C, $R C_{n}^{j}$ - actual answer (Response) to question $n$ given by participant $j$ from Group C, NC - Number of participants in group C (32 participants).

The last column presents, for each category of participants, the statistics for the hypothesis that the mean of the personal anchoring measures is greater than zero, which is consistent with the respective category of participants on average exhibiting anchoring bias.

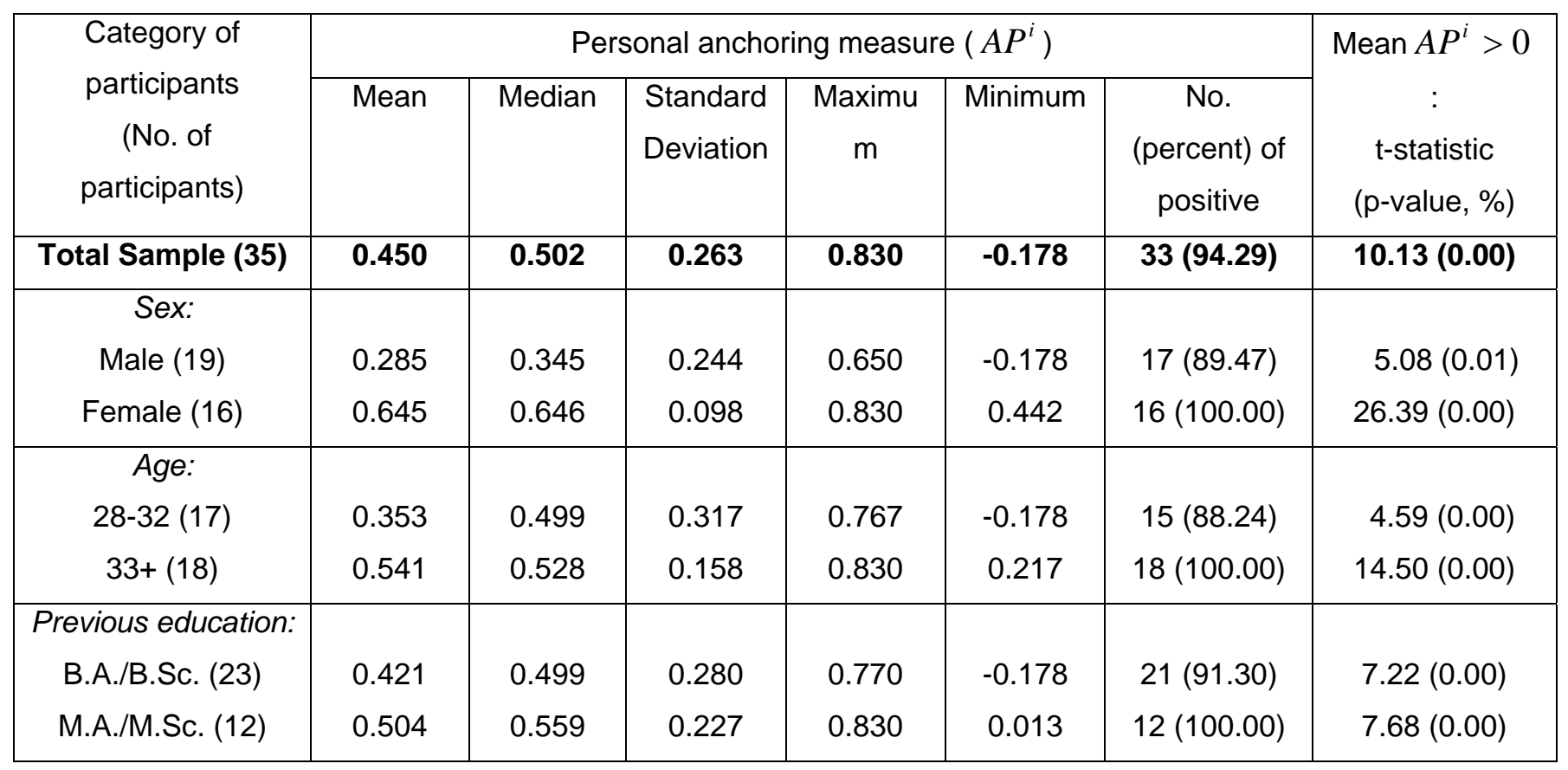


Table 3

Mean anchoring measure statistics, for "easy" and "difficult" questions

The table reports, by groups of questions, summary statistics of the mean anchoring measures $\left(A Q_{n}\right)$ calculated for each question as follows:

$A Q_{n}=\frac{\sum_{i=1}^{N H} A_{i}^{n}}{N A}$ with $A_{n}^{i}=1-\frac{\left\|R A_{n}^{i}-I_{n}\right\|}{D C_{n}}$ and $D C_{n}=\frac{\sum_{j=1}^{N C}\left\|R C_{n}^{j}-I_{n}\right\|}{N C}$

Where: $A Q_{n}$ - mean Anchoring measure for Question $n, N A$ - Number of participants in group A (35 participants), $A_{n}^{i}$ - Anchoring (bias) measure for question $n$ and person $i, R A_{n}^{i}$ - actual answer (Response) to question $n$ given by participant $i$ from Croup $A, I_{n}$ - anchor Indicator (anchor) for question $n, D C_{n}$ - mean Deviation from the anchor for question $n$, in Group C, $R C_{n}^{j}$ - actual answer (Response) to question $n$ given by participant $j$ from Group C, NC - Number of participants in group C (32 participants).

The last row reports statistics for the tests of equality of means and medians between the "easy" and the "difficult" questions.

\begin{tabular}{|c|c|c|c|c|c|c|}
\hline \multirow{2}{*}{$\begin{array}{c}\text { Category of questions } \\
\text { (No. of questions) }\end{array}$} & \multicolumn{6}{|c|}{ Mean anchoring measure $\left(A Q_{n}\right)$} \\
\cline { 2 - 7 } & Mean & Median & $\begin{array}{c}\text { Standard } \\
\text { Deviation }\end{array}$ & Maximum & Minimum & $\begin{array}{c}\text { No. (percent) of } \\
\text { positive }\end{array}$ \\
\hline Total Sample (21) & $\mathbf{0 . 4 5 0}$ & $\mathbf{0 . 4 2 2}$ & $\mathbf{0 . 2 1 1}$ & $\mathbf{0 . 7 4 4}$ & $\mathbf{0 . 0 4 2}$ & $\mathbf{2 1 ~ ( 1 0 0 . 0 0 )}$ \\
\hline "Easy" (11) & 0.341 & 0.378 & 0.201 & 0.641 & 0.042 & $11(100.00)$ \\
"Difficult" (10) & 0.569 & 0.570 & 0.155 & 0.744 & 0.396 & $10(100.00)$ \\
\hline $\begin{array}{c}\text { Tests of equalitya: } \\
\text { Stat. value (p-value, } \\
\%)\end{array}$ & $\mathbf{2 . 8 9 ( 0 . 9 3 )}$ & $\mathbf{2 . 7 8 ( 0 . 5 4 )}$ & & & & \\
\end{tabular}

a We employ t-test for the equality of means between series, and Wilcoxon/Mann-Whitney test for the equality of medians between series. 
Table 4

Am. J. Soc. Mgmt. Sci., 2010, 1(2): 164-180

\section{Mean personal anchoring measure statistics, for "easy" and "difficult" questions}

The table reports, by categories of participants, summary statistics of the personal anchoring measures calculated separately for "easy" ( $\left.A P E^{i}\right)$ and "difficult" $\left(A P D^{i}\right)$ questions, for each of the participants in Group A:

$A P E^{i}=\frac{\sum A_{i}^{n} \mid \text { easy }}{11}$ and $A P D^{i}=\frac{\sum A_{i}^{n} \mid \text { difficult }}{10}$

with $A_{n}^{i}=1-\frac{\left\|R A_{n}^{i}-I_{n}\right\|}{D C_{n}}$ and $D C_{n}=\frac{\sum_{j=1}^{N C}\left\|R C_{n}^{j}-I_{n}\right\|}{N C}$

Where: $A P E^{i}$ - mean Personal Anchoring measure for "Easy" questions for participant $i, A P D^{i}$ - mean Personal Anchoring measure for "Difficult" questions for participant $i, \sum A_{i}^{n} \mid$ easy - sum of participant i's Anchoring measures for "easy" questions, $\sum A_{i}^{n} \mid$ difficult - sum of participant i's Anchoring measures for "difficult" questions, $A_{n}^{i}$ - Anchoring (bias) measure for question $n$ and person $i, R A_{n}^{i}$ - actual answer (Response) to question $n$ given by participant $i$ from Croup A, $I_{n}$ - anchor Indicator for question $n, D C_{n}$ - mean Deviation from the anchor for question $n$, in Group C, $R C_{n}^{j}$ - actual answer (Response) to question $n$ given by participant $j$ from Group C, $N C$ - Number of participants in group C (32 participants). The last row of each block reports statistics for the tests of equality of means and medians between "easy" and "difficult" questions, for subsamples of participants.

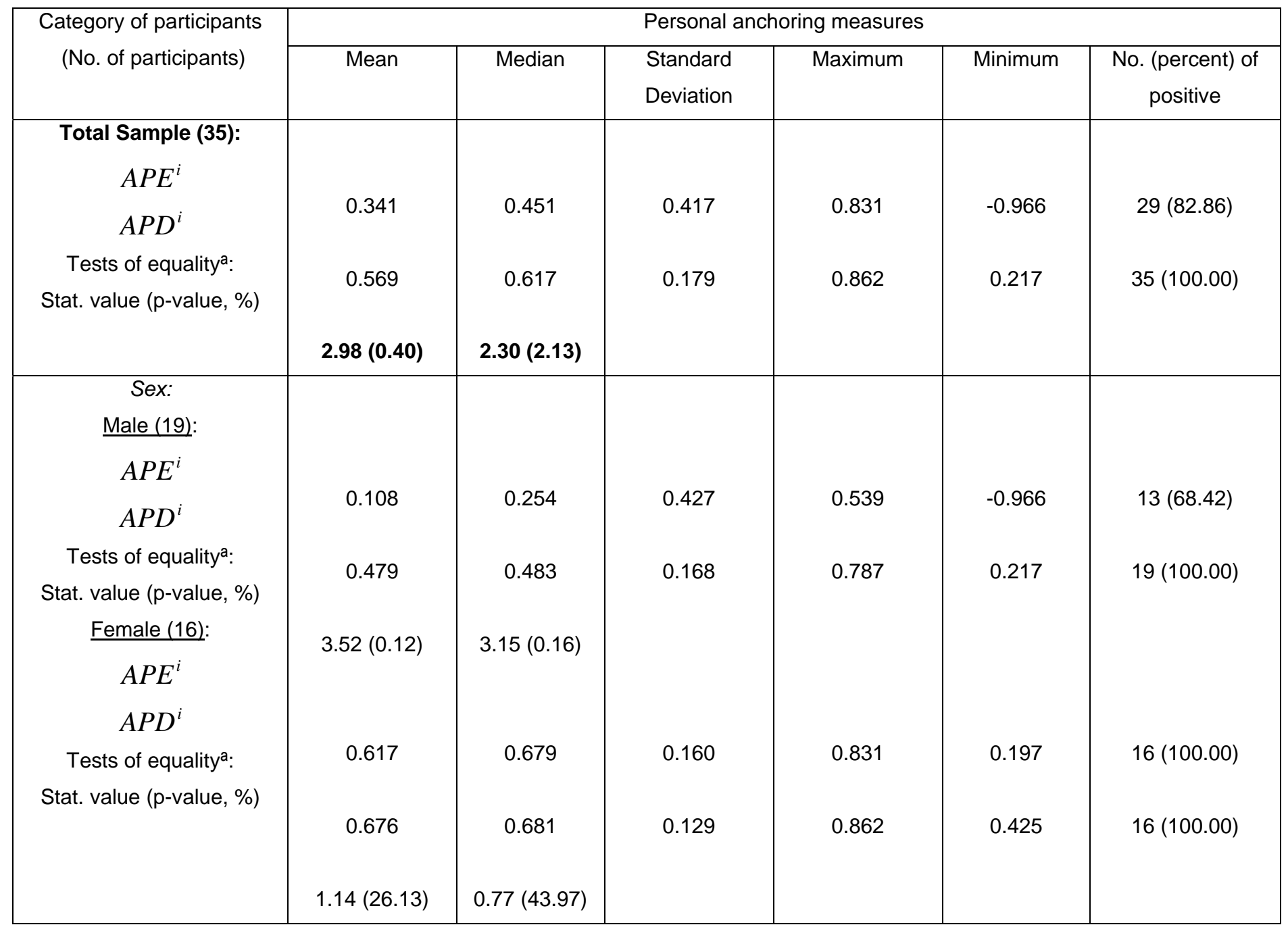


Table 4 (continued)

Am. J. Soc. Mgmt. Sci., 2010, 1(2): 164-180

\begin{tabular}{|c|c|c|c|c|c|c|}
\hline \multirow{2}{*}{$\begin{array}{l}\text { Category of participants } \\
\text { (No. of participants) }\end{array}$} & \multicolumn{6}{|c|}{ Personal anchoring measures } \\
\hline & Mean & Median & $\begin{array}{l}\text { Standard } \\
\text { Deviation }\end{array}$ & Maximum & Minimum & $\begin{array}{l}\text { No. (percent) of } \\
\text { positive }\end{array}$ \\
\hline \multicolumn{2}{|l|}{$\underline{28-32(17):}$} & & & & & \\
\hline$A P E^{i}$ & 0.176 & 0.370 & 0.522 & 0.709 & -0.966 & $11(64.71)$ \\
\hline$A P D^{i}$ & 0.545 & 0.546 & 0.185 & 0.862 & 0.227 & $17(100.00)$ \\
\hline Tests of equalitya: & & & & & & \\
\hline Stat. value ( $p$-value, \%) & $2.74(1.00)$ & $1.83(6.79)$ & & & & \\
\hline \multicolumn{7}{|l|}{ 33+ (18): } \\
\hline$A P E^{i}$ & 0.495 & 0.453 & 0.200 & 0.831 & 0.197 & $18(100.00)$ \\
\hline$A P D^{i}$ & 0.592 & 0.632 & 0.176 & 0.842 & 0.217 & $18(100.00)$ \\
\hline \multicolumn{7}{|l|}{ Tests of equalitya: } \\
\hline \multirow[t]{2}{*}{ Stat. value ( $p$-value, \%) } & $1.55(13.13)$ & 1.34 & & & & \\
\hline & & $(17.87)$ & & & & \\
\hline \multirow{2}{*}{\multicolumn{7}{|c|}{$\begin{array}{l}\text { Previous education: } \\
\text { B.A./B.Sc. (23): }\end{array}$}} \\
\hline & & & & & & \\
\hline$A P E^{i}$ & 0.305 & 0.451 & 0.444 & 0.754 & -0.966 & 19 (82.61) \\
\hline$A P D^{i}$ & 0.549 & 0.617 & 0.189 & 0.862 & 0.217 & $23(100.00)$ \\
\hline Tests of equalitya: & & & & & & \\
\hline Stat. value (p-value, \%) & $2.42(1.97)$ & $1.80(7.16)$ & & & & \\
\hline \multicolumn{7}{|l|}{ M.A./M.Sc. (12): } \\
\hline$A P E^{i}$ & 0.409 & 0.476 & 0.368 & 0.831 & -0.412 & $10(83.33)$ \\
\hline$A P D^{i}$ & 0.609 & 0.585 & 0.159 & 0.828 & 0.358 & $12(100.00)$ \\
\hline Tests of equality ${ }^{\mathrm{a}}$ : & & & & & & \\
\hline Stat. value (p-value, \%) & $1.72(9.87)$ & 1.30 & & & & \\
\hline & & (19.39) & & & & \\
\hline
\end{tabular}

a We employ t-test for the equality of means between series, and Wilcoxon/Mann-Whitney test for the equality of medians between series. 
Am. J. Soc. Mgmt. Sci., 2010, 1(2): 164-180

\section{Appendix B: Research questionnaire (Group A)}

Thank you for taking part in the experiment!

Please, don't consult your colleagues while answering the questions. The questionnaire is anonymous and is intended for research purposes only.

Below you will find a number of real financial data. Please fill in the missing numbers, according to your best estimation.

1. The current value of S\&P 500 Index is 1172 points.

I suppose that the current value of TA- $25^{10}$ Index is

2. The S\&P 500 Index annual return at 2009 was $23.45 \%$.

I suppose that the TA-25 Index annual return at 2009 was $\%$.

3. The average annual return of S\&P 500 Index over the years $2007-2009$ was- $-7.70 \%$.

I suppose that the average annual return of TA25 Index over the years 2007-2009 was $\%$.

4. The current value of S\&P 500 Index differs from the Index's historical high by $\underline{25.11 \%}$.

I suppose that the current value of TA25 Index differs from the Index's historical high by $\%$.

5. The manufacturing production in Israel increased in 2008 relatively to 2007 by $\underline{7.39 \%}$.

I suppose that the TA-100 11 Index annual return at 2009 was $\%$.

6. The average annual rate of increase in the manufacturing production in Israel over the years 19982008 was $\underline{3.55 \%}$.

I suppose that the average annual return of TA-100 Index over the years 2007-2009 was $\%$.

7. The major increase in the manufacturing production in Israel in the last decade took place in 2000.

The manufacturing production increased by $\underline{9.88 \%}$.

I suppose that the current value of TA-100 Index differs from the Index's historical high by $\%$.

8. The DAX Index annual return at 2009 was $\underline{23.85 \%}$.

I suppose that the Mid-Cap-50 (Yeter 50) Index ${ }^{12}$ annual return at 2009 was $\%$.

9. The current value of DAX Index differs from the Index's historical high by $\underline{23.71 \%}$.

I suppose that the current value of Mid-Cap-50 (Yeter 50) Index differs from the Index's historical high by $\%$.

10. The agricultural output in Israel increased in 2008 relatively to 2007 by $\underline{7.45 \%}$.

I suppose that the Tel-Bond 20 Index $^{13}$ annual return at 2009 was $\%$.

\footnotetext{
10 Index that tracks the prices of the shares of the 25 companies with the highest market capitalization on the Tel Aviv Stock Exchange.

11 Index that consists of the 100 stocks with the highest market capitalization on the Tel Aviv Stock Exchange.

12 Index that includes 50 stocks with the highest market capitalization that are not included in the TA-100 Index.
} 
11. The agricultural output in Israel increased in 2008 relatively to 1998 by $\underline{16.28 \%}$.

I suppose that the current before taxes 5-years yield to maturity on Galil (CPI-linked fixed rate) government bonds is equal to $\%$.

12. The major increase in the agricultural output in Israel in the last decade took place in 2004 . The agricultural output increased by $\underline{10.1 \%}$.

I suppose that the current before taxes 5-years yield to maturity on Shahar (non-linked fixed rate) government bonds is equal to $\%$.

13. The inflation rate in the US in from the beginning of this year is $\underline{1.8 \%}$.

I suppose that the inflation rate in Israel from the beginning of this year is $\%$.

14. The inflation rate in the US in 2009 was $\underline{2.7 \%}$.

I suppose that the inflation rate in Israel in 2009 was $\%$.

15. The average annual inflation rate in the US over the years 2007-2009 was $2.29 \%$.

I suppose that the average annual inflation rate in Israel over the years 2007-2009 was $\%$.

16. The gross domestic product of Israel increased in 2008 relatively to 2007 by $\underline{2.2 \%}$.

I suppose that the current Interest Rate of Bank of Israel is equal to $\%$.

17. The gross domestic product of Israel increased in 2006 relatively to 2005 by $\underline{3.4 \%}$.

I suppose that the current Prime Interest Rate ${ }^{14}$ is equal to $\%$.

18. The Yen/Shekel exchange rate changed from the beginning of this year by $-0.45 \%$.

I suppose that the Dollar/Shekel exchange rate changed from the beginning of this year by $\%$.

19. The Yen/Shekel exchange rate changed over the year 2009 by $\underline{-3.66 \%}$.

I suppose that the Dollar/Shekel exchange rate changed over the year 2009 by $\%$.

20. The Dollar-to-British Pound exchange rate (Dollars for 1 Pound) changed from the beginning of this year by $\underline{-6.67 \%}$.

I suppose that the Euro/Shekel exchange rate changed from the beginning of this year by $\%$.

21. The Dollar-to-British Pound exchange rate (Dollars for 1 Pound) changed over the year 2009 by = 9.13\%.

I suppose that the Euro/Shekel exchange rate changed over the year 2009 by $\%$.

\footnotetext{
${ }^{13}$ Index that consists of the 20 corporate bonds, fixed-interest and CPI-linked, with the highest market capitalization among all the bonds traded on the Tel-Aviv Stock Exchange.

14 Quoted basic interest rate fixed by large commercial banks. In the last years, in Israel, it is actually equal to the Interest Rate of the Bank of Israel plus 1.5\%.
} 